\title{
Automatic Assume Guarantee Analysis for Assertion-Based Formal Verification
}

\author{
Dong Wang ${ }^{\dagger}$ \\ Synopsys Inc. \\ dongw@synopsys.com
}

\author{
Jeremy Levitt ${ }^{\dagger}$ \\ Mentor Graphics Corp. \\ jeremy_levitt@mentorg.com
}

\begin{abstract}
Assertion based verification encourages the insertion of many assertions into a design. Typically, not all assertions can be proven (or falsified). The indeterminate assertions require manual analysis in order to determine design correctness a time-consuming and error-prone process. This paper shows how automatic assume guarantee reasoning can be used to reduce the amount of manual analysis. We present algorithms to automatically compute the assume guarantee relations between assertions. We extend circular assume guarantee reasoning to compute more proofs. And, we show how automatic assume guarantee reasoning can be used in practice to reduce the number of indeterminate assertions requiring manual analysis. We present the results of applying our algorithms to large industrial designs.
\end{abstract}

\section{INTRODUCTION}

Assertion based verification (ABV) is a methodology for verifying correct design implementation. Assertions, in the form of safety properties, are added to the design as it is being coded. These assertions check for the correct behavior of the logic being coded as well as for correct behavior on the design interfaces. As the design and verification evolve, the number of assertions can become very large. It is typical for a design block to have hundreds of assertions. It is also typical that a considerable number of assertions are too difficult to be formally proven or falsified within the given resource limits. For crucial assertions, such as bus mutual exclusion (mutex) properties and logic equivalence checking (LEC) constraints, etc., manual inspection of each indeterminate assertion is frequently mandated. The consequence of these assertions failing is likely to be a completely non-functional chip. Manual inspection is clearly a tedious, time-consuming and error-prone process. Therefore, it is desirable to reduce the number of assertions that require manual analysis.

This paper describes automatic assume guarantee algorithms designed to exploit the high assertion density of ABV. Our algorithms automatically compute the assume guarantee relations between given sets of assertions. We exploit the automatically computed assume guarantee relations in two ways. Firstly, we use the assume guarantee relations to generate additional proofs. To increase the number of proofs obtained, we present an algorithm that extends circular assume guarantee reasoning to compute additional proofs. Secondly, we use the assume guarantee relations between the indeterminate

\footnotetext{
${ }^{\dagger}$ This work was done while the authors were at 0-In Design Automation.
}

assertions to reduce the number of indeterminate assertions that require manual analysis. An assume guarantee relation states that an assertion is implied (guaranteed) if a set of assumed assertions are valid. Since the correctness of the assertion is implied by a set of assumed assertions, only the assumed assertions need to be analyzed manually. We present a practical and simple method to organize this information so that fewer assertions need be manually analyzed.

\section{A. Related work}

The assume guarantee algorithms we present in this paper are different in important respects from those proposed by previous research. Most existing assume guarantee algorithms are manual [10] [9] [7]. Users are required to identify the assume guarantee relations between properties, and the algorithms then check the validity of the given relations and use them to generate proof obligations. In contrast, our algorithms automatically compute assume guarantee relations by enhancing existing SAT based temporal induction and abstraction refinement algorithms. Recently there has been some interesting research on automating assume guarantee, such as [1] [4]. [1] [4] focus on automatically synthesizing a suitable environment to guarantee a property, but this synthesis can be prohibitively expensive to perform. In contrast, our algorithms are designed for $\mathrm{ABV}$ where the number of assertions is usually large. Instead of constructing completely new assumptions, our algorithms efficiently identify useful assumptions within the set of user specified assertions.

Most previous research on assume guarantee is directed at generating more proofs. In this paper, the assume guarantee relations are also used to reduce the manual verification effort involved when assertions can not be formally proven or falsified. Other previous research uses dependencies between assertions to avoid redundant error reporting when design bugs are found. Our approach on the other hand, uses assume guarantee relations to help users verify their design even when no design bugs have been found yet.

\section{B. Contributions}

We have designed several new assume guarantee algorithms to not only compute proofs, but just as importantly to reduce the manual verification effort required for indeterminate assertions. 
With $\mathrm{ABV}$, it is impractical for users to specify the assume guarantee relations between assertions. Designers and verification engineers have neither the knowledge nor the inclination to perform such analysis. Thus, we show how to enhance existing SAT based temporal induction and abstraction refinement algorithms to automatically compute assume guarantee relations. Our algorithms are based on SAT unsatisfiability proofs and abstract counterexample simulation. Similar to the use of constraints, assumptions are used to strengthen induction hypothesis for temporal induction, and to generate smaller abstractions for abstraction refinement.

We present an algorithm, called Guarantee, that uses the automatically computed assume guarantee relations to deduce guaranteed assertions from a given arbitrary set of assumed assertions. The traditional application of the circular assume guarantee rule [9] (described in Section II-B) is a special case of our algorithm, specifically when (1) the given set of assumed assertions is empty and (2) each property is implied by a single set of assumptions. Our algorithm allows a nonempty set of assumed assertions, enabling the deduction of general dependencies between assertions (in addition to proofs deduced from the empty set of assumed assertions). Our algorithm handles multiple sets of assumptions per property and this enables proofs to be deduced in the presence of zerodelay cycles (described in Section II-B).

We also present an algorithm, called Guide, that distills the information represented by all the automatically computed assume guarantee relations into an organized and actionable format, thereby reducing the manual effort required to analyze the indeterminate assertions. This is of practical importance, since a direct presentation of all the assume guarantee relations is more complicated than most designers and verification engineers are prepared to accept: the number of properties having assumptions can be large, an assumption of a property can itself have assumptions, the assume guarantee relations may contain loops, and there are different assumption types (zero-delay or unit-delay) to keep track of.

\section{Organization}

The remainder of the paper is organized as follows. Section II introduces notation and the circular assume guarantee rule. Section III describes automatic algorithms to compute assume guarantee relations. Section IV describes the Guarantee algorithm to compute guarantees for an arbitrary set of assertions. Section V describes the Guide algorithm to generate organized and actionable assume guarantee reports. Section VI presents experimental results. Finally, Section VII contains conclusions.

\section{BACKGROUND}

\section{A. Notations}

In this paper, the design under verification is modeled by a machine $M=\langle F, X, I, R, P\rangle$, where $F$ is the set of primary inputs, $X$ is the set of registers, $I(X)$ is the initial state predicate, $R\left(X, F, X^{\prime}\right)$ is the next state predicate, and $P \subseteq X$ is the set of assertions. An assertion $p \in P$ is true under $M$ if and only if $M \models \square p$, i.e. $p$ holds in all reachable states of $M$. In bounded model checking [2] (BMC) and temporal induction [11], machine $M$ is unrolled to different times. Let $F^{k}$ and $X^{k}$ be the unrolled input and register variables at time $k$. Given a set of properties $C \subseteq P$, a single property $p$ and an unroll $k$, the BMC and temporal induction for $p$ under constraints $C$ are defined as:

$$
\begin{aligned}
\mathrm{BMC}_{k}(C, p)= & I\left(X^{0}\right) \wedge R\left(X^{0}, F^{0}, X^{1}\right) \wedge \\
& C\left(X^{1}\right) \wedge R\left(X^{1}, F^{1}, X^{2}\right) \wedge \cdots \wedge \\
& C\left(X^{k-1}\right) \wedge R\left(X^{k-1}, F^{k-1}, X^{k}\right) \wedge \\
& C\left(X^{k}\right) \wedge \neg p^{k} \\
\operatorname{IND}_{k}(C, p)= & C\left(X^{0}\right) \wedge p^{0} \wedge R\left(X^{0}, F^{0}, X^{1}\right) \wedge \cdots \wedge \\
& C\left(X^{k-1}\right) \wedge p^{k-1} \wedge R\left(X^{k-1}, F^{k-1}, X^{k}\right) \wedge \\
& C\left(X^{k}\right) \wedge \neg p^{k}
\end{aligned}
$$

\section{B. Circular Assume Guarantee Reasoning}

Given a machine $M$, a property $p \in P$ and two disjoint sets of properties $A_{p}^{+} \subseteq P$ and $A_{p}^{0} \subseteq(P \backslash\{p\})$, circular assume guarantee reasoning [9] deals with triples of the form $\left\langle\left\langle A_{p}^{+}, A_{p}^{0}\right\rangle, M, p\right\rangle$, which asserts that in machine $M$, assuming $A_{p}^{+}$up to time $t-1$ and $A_{p}^{0}$ up to time $t$ can deduce $p$ up to time t. $A_{p}^{+}$is called the unit-delay assumptions for $p$, and $A_{p}^{0}$ is the set of zero-delay assumptions for $p$. We will use $A_{p}=A_{p}^{+} \cup A_{p}^{0}$ to denote all the assumptions for $p$, and $\left\langle A_{p}, M, p\right\rangle$ to denote the triple. We say $p$ is implied by assumptions $A_{p}$. With zero delay assumptions, care must be taken to avoid false "proofs". For example if two false assertions are equivalent, each assertion is implied by the other, yet neither is true. Circular assume guarantee reasoning requires non-zero-delay (i.e. unit-delay) cycles to generate proofs.

Formally, $M \models \square\left(\wedge_{p_{i} \in P} p_{i}\right)$, if

- every property $p_{i}$ is implied by assumptions, i.e. $\left\langle\left\langle A_{p_{i}}^{+}, A_{p_{i}}^{0}\right\rangle, M, p_{i}\right\rangle$,

- and there exists a well founded order $\preceq$ on $P$, such that $\forall q \in A_{p_{i}}^{0}\left(q \preceq p_{i}\right)$.

For formal hardware verification of safety properties without fairness constraints, proving $\left\langle\left\langle A_{p_{i}}^{+}, A_{p_{i}}^{0}\right\rangle, M, p_{i}\right\rangle$ is reduced to an induction problem or a reachability problem (see Section III for details).

During verification, each property $p \in P$ can be in one of four possible states representing the current verification result: unknown, true, false or implied. Initially all properties are unknown. A property becomes true or false when we find either a proof or counterexample respectively for it. A property becomes implied when we find a set of assumptions that imply it. We use $P_{\text {unknown }}, P_{\text {true }}, P_{\text {false }}, P_{\text {impld }}$ to represent the corresponding set of assertions. As discussed, we allow a single property $p$ to have more than one set of assumptions $A_{p, j}$, where $1 \leq j \leq k_{p}$ and $\left\langle A_{p, j}, M, p\right\rangle \wedge\left(A_{p, j} \cap P_{\text {true }}=\right.$ $\emptyset) \wedge\left(A_{p, j} \cap P_{\text {false }}=\emptyset\right)$. We use $A_{p}$ to denote any of the valid set of assumptions for $p$. Whenever a property $q \in A_{p}$ is proven, it is removed from $A_{p}$. If this makes $A_{p}$ empty, $p$ is proven to be true. On the other hand, if $q$ is shown to be false, $A_{p}$ is no longer a valid set of assumptions for $p$ and the 
entire set is removed. If it is the only set of assumptions for $p$, then $p$ becomes unknown. Our algorithms may also directly prove or falsify $p$. A property is indeterminate if it is either unknown or implied.

For a set of properties $C \subseteq P$, we use $M \models \square C$ as a shorthand for $M \models \square\left(\wedge_{c_{i} \in C} c_{i}\right)$. We say $p$ is guaranteed by $C$ if and only if $M \models(\square C \rightarrow \square p)$. Intuitively, if $C$ is true under $M$ then any guaranteed property must be true under $M$. It is informative to note that $\langle C, M, p\rangle$ is a stronger statement than $M \models(\square C \rightarrow \square p)$. For example, let $M=$ $\langle\emptyset,\{p, q\}, I, R,\{p, q\}\rangle$, such that:

$$
\begin{aligned}
I(p)=I(q) & =1 \\
R(p) & =0 \\
R(q) & =p
\end{aligned}
$$

It is easy to see that the only trace of $M$ is:

\begin{tabular}{|l|l|l|}
\hline time & $\mathbf{p}$ & $\mathbf{q}$ \\
\hline \hline$t=0$ & 1 & 1 \\
\hline$t=1$ & 0 & 1 \\
\hline$t \geq 2$ & 0 & 0 \\
\hline
\end{tabular}

Thus, $M \models \square q \rightarrow \square p$ holds because there is no path where $q$ holds globally. $\langle\langle\emptyset,\{q\}\rangle, M, p\rangle$ on the other hand does not hold, because at time $t=1$, property $q$ is 1 , but $p$ is 0 and so $q$ cannot imply $p$.

\section{IDENTIFY ASSUMPTIONS}

In this section, we show how to enhance existing formal verification algorithms to compute assumptions automatically.

\section{A. Temporal Induction}

SAT based temporal induction [11] [5] complements BMC by establishing an upper bound on the length of the shortest counterexample. This bound is typically much smaller than other bounds, such as diameter or recurrence diameter. However, as is well known, temporal induction often fails due to inadequate induction hypotheses. We use the following simple SAT based verification algorithm as the basis of our enhancement.

$/ / \mathrm{m}$ is the given bound

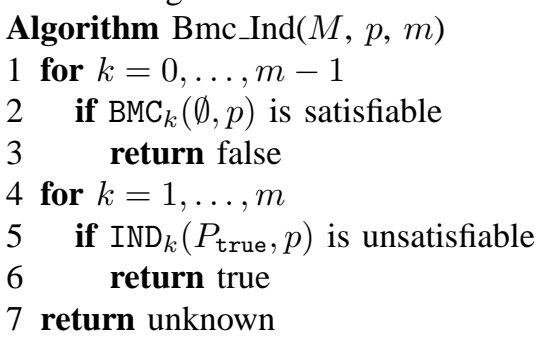

Fig. 1. Bounded model checking and temporal induction

Let $\overline{P_{\text {false }}}=P \backslash P_{\text {false }}$. Next we show how to use $\overline{P_{\text {false }}}$ to strengthen induction without sacrificing soundness. The following example is used to motivate our approach. Let machine $M$ have 8-bit inputs $i n_{1}, i n_{2}, 8$-bit registers cntr $_{1}, c n t r_{2}$, and property registers $w_{1}, w_{2}$. The initial state and next state functions are:

$$
\begin{array}{rll}
I\left(\text { cntr }_{1}\right) & =17 \\
I\left(\text { cntr }_{2}\right) & =13 \\
R\left(\text { cntr }_{1}\right) & =w_{1}==w_{2} ? & \\
& & \begin{array}{l}
\text { cntr }_{1} * 3-i n_{1} * 2: \\
\text { cntr }_{1}+i n_{1}
\end{array} \\
R\left(\text { cntr }_{2}\right) & =w_{1}==w_{2} ? & \begin{array}{l}
\text { cntr }_{2} * 5-i n_{2} * 2: \\
\text { cntr }_{2}+i n_{2}
\end{array} \\
I\left(w_{1}\right) & =1 & \\
I\left(w_{2}\right) & =1 & \\
R\left(w_{1}\right) & =\text { cntr }_{1}[0] & \\
R\left(w_{2}\right) & =\text { cntr }_{2}[0] &
\end{array}
$$

It is easy to see that the two assertions $w_{1}, w_{2}$ are true since values in cntr $_{1}$ and cntr $_{2}$ are always odd numbers. But, algorithm Bmc_Ind fails to prove $w_{1}$ and $w_{2}$ regardless of the induction depth. The induction, $\operatorname{IND}_{k}(C, p)$, can be easily falsified because it ignores the initial state of the machine. For example, Table I shows a failed induction for $w_{1}$ with 4 unrolls. However, if both $w_{1}$ and $w_{2}$ at time $t-1$ and time $t$

\begin{tabular}{|l|r|r|r|r|r|r|}
\hline time & $w_{1}$ & cntr $_{1}$ & in $_{1}$ & $w_{2}$ & cntr $_{2}$ & in $_{2}$ \\
\hline \hline 0 & 1 & 1 & 2 & 0 & 0 & 0 \\
\hline 1 & 1 & 3 & 2 & 0 & 0 & 0 \\
\hline 2 & 1 & 5 & 1 & 0 & 0 & 0 \\
\hline 3 & 1 & 6 & 0 & 0 & 0 & 0 \\
\hline 4 & 0 & 6 & 0 & 0 & 0 & 0 \\
\hline
\end{tabular}

TABLE I

INDUCTION COUNTEREXAMPLE FOR $w_{1}$

are assumed, we can easily prove $w_{1}$ at time $t+1$ and similarly for $w_{2}$. Based on the circular rule in Section II-B, both $w_{1}$ and $w_{2}$ are proven.

Figure 2 shows the modifications of Line 4 to Line 6 in Figure 1. For each unroll $k$, when proving $p$ we use as

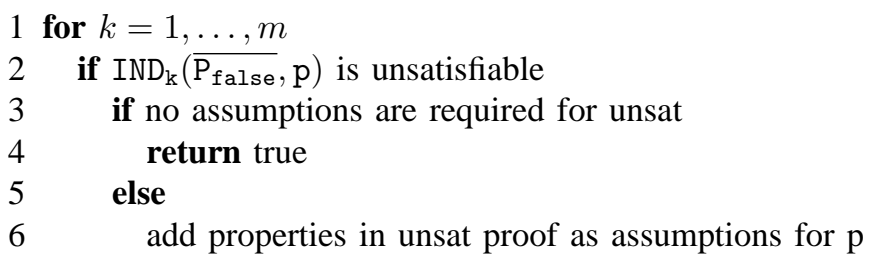

Fig. 2. Temporal induction with assumptions

constraints all the properties not known to be false. In the case of unsatisfiability, we extract the unsatisfiable core [3] from the SAT solver (Line 2). Let $U=\left\{p_{i}^{j} \mid\left(p_{i} \neq p\right) \wedge\left(p_{i}^{j} \in\right.\right.$ unsat_core $\left.) \wedge p_{i} \in\left(P_{\text {unknown }} \cup P_{\text {impld }}\right)\right\}$ be a subset of property variables that appear in the unsatisfiable core. $U$ is the set of assumed assertions responsible for the success of the inductive hypothesis. If $U$ is empty (Line 3), $p$ is proven without assumptions and the algorithm terminates with a proof (Line 4). Otherwise, an assume guarantee relation $\left\langle\left\langle A_{p}^{+}, A_{p}^{0}\right\rangle, M, p\right\rangle$, where $A_{p}^{0}=\left\{p_{i} \mid p_{i}^{k} \in U\right\}$ and $A_{p}^{+}=\left\{p_{i} \mid p_{i} \notin A_{p}^{0} \wedge \exists j<\right.$ 
$\left.k\left(p_{i}^{j} \in U\right)\right\}$ has been computed. Even if $p$ is implied by assumptions at unroll $k$ (Line 6), it may be possible to prove $p$ at a larger unroll using fewer or no assumptions because larger unrolls will apply constraints in more time frames. In general, proofs from SAT solvers are highly affected by the state of the SAT database and SAT heuristics used. We purposely vary the decision heuristics. As a result, assumptions identified in different unrolls may not subsume each other. We record every set of assumptions for each property $p$, unless it contains an existing set of assumptions or $p$ is proven without assumptions.

\section{B. Abstraction Refinement}

Existential abstraction refinement is an effective technique for combating the state explosion problem associated with model checking. The objective of abstraction-refinement algorithms is to generate a small abstraction that both preserves the validity of a property and is amiable to direct model checking. We show how assumptions can be used to help reduce the size of these abstractions.

We first introduce a BDD based reachability algorithm which computes $\left\langle A_{p}, M, p\right\rangle$ and separates the unit-delay assumptions in $A_{p}$ from the zero-delay assumptions in $A_{p}$. The algorithm is based on the standard forward reachability algorithm:

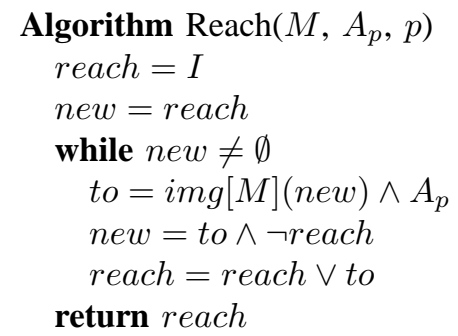

Essentially, reachability is restricted to the set of states that satisfy all properties in $A_{p} .\left\langle A_{p}, M, p\right\rangle$ holds when the reachable states do not violate $p$. Zero-delay assumptions are identified during reachability when $p$ is violated in a state $s$ that is excluded because $s$ also violates $q \in A_{p}$. All the other assumptions are unit-delay assumptions.

We have enhanced the counterexample guided localization reduction algorithm in [12], and the proof-based without counterexample algorithm in [8], [6] to identify useful assumptions. The refinement algorithm in [12] is based on simulating the abstraction counterexamples on the concrete machines using 3 -value simulation. Our enhancement of this algorithm is very simple. A property $q \in \overline{P_{\text {false }}}$ is added to the abstraction, whenever the 3 -value simulation value for $q$ becomes 0 . Therefore by assuming $q$, the abstract counterexample is invalidated.

Abstraction without counterexamples [8] [6] uses unsatisfiable BMC instances to construct abstraction. The circuit nodes that appear in the unsatisfiable core of $\mathrm{BMC}_{k}(\emptyset, p)$ are extracted and model checked to see whether they are also valid for an unbounded proof. Our enhancement is to create a smaller abstraction based on the unsatisfiability proof of $\mathrm{BMC}_{k}\left(\overline{P_{\text {false }}}, p\right)$. Assuming that the properties in $\overline{P_{\text {false }}}$ are impossible to violate in time frames 1 to $k$, the two SAT problems $\operatorname{BMC}_{k}(\emptyset, p)$ and $\mathrm{BMC}_{k}\left(\overline{P_{\text {false }}}, p\right)$ are equivalent in that they are both unsatisfiable. However, the size of the unsatisfiability core for the latter can be much smaller than the former. A trivial example is $\mathrm{BMC}_{k}(\{p\}, p)$, which does not use any circuit information to determine unsatisfiability.

\section{Compute Guarantees}

In this section, we show how to compute guaranteed assertions for a given arbitrary set of assumptions using the computed assume guarantee relations. Our new algorithm is based on a proof graph similar to an AND-OR graph:

Definition 4.1: A proof graph $\mathcal{G}$ is a directed cyclic graph with 5-tuple $\left\langle\mathcal{T}, \mathcal{A}, \mathcal{O}, \mathcal{E}, \mathcal{E}^{+}\right\rangle$, where

- $\mathcal{T}$ is the set of terminal vertices. Each property $p \in$ $P_{\text {unknown }} \cup P_{\text {impld }}$ has a corresponding terminal vertex $\tau_{p} \in \mathcal{T}$.

- $\mathcal{A}$ is a set of AND vertices. Each set of assumptions $A_{p, j}$ for $p \in P_{\text {impld }}$ corresponds to an AND vertex $\alpha_{p}^{j}$. For each assumption $q \in A_{p, j}$, there is an edge $\left\langle\tau_{q}, \alpha_{p}^{j}\right\rangle \in \mathcal{E}$.

- $\mathcal{O}$ is a set of OR vertices. Each implied property $p \in$ $P_{\text {impld }}$ has a corresponding OR vertex $o_{p}$. There is an edge $\left\langle o_{p}, \tau_{p}\right\rangle \in \mathcal{E}$. If $p$ has $k_{p}$ sets of assumptions, there are $k_{p}$ edges $\left\langle\alpha_{p}^{j}, o_{p}\right\rangle \in \mathcal{E}$, for $j \in\left\{1, \ldots, k_{p}\right\}$.

- $\mathcal{E}^{+}=\left\{\left\langle\tau_{q}, \alpha_{p}^{j}\right\rangle \mid q \in A_{p, j}^{+}\right\}$represents the unit-delay edges in $\mathcal{E}$. All the other edges in $\mathcal{E}$ have zero-delay.

We also let $\mathcal{V}=\{\mathcal{T} \cup \mathcal{A} \cup \mathcal{O}\}$.

For example, Figure 3 shows a proof graph. In this graph, there

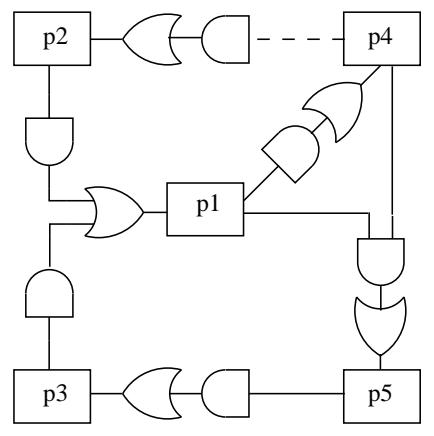

Fig. 3. An example proof graph

are 5 implied properties $p_{1}$ to $p_{5}$. The dashed line between $p_{4}$ and $p_{2}$ represents a unit-delay edge; all the other edges have zero-delay. $p_{1}$ is guaranteed assuming either $p_{2}$ or $p_{3}$. Thus $p_{1}$ has two sets of assumptions. $p_{5}$ is guaranteed if $p_{1}$ and $p_{4}$ both assumed. Based on model checking results, we know there is a $t$, such that all five properties hold from time 0 to time $t-1$. From Figure 3 , we have

$$
\begin{aligned}
p_{4}^{t-1} \rightarrow p_{2}^{t} \rightarrow p_{1}^{t} & \rightarrow p_{4}^{t} \\
& \downarrow \\
& p_{5}^{t} \rightarrow p_{3}^{t}
\end{aligned}
$$

Therefore, by induction all five properties are true. Although there is a zero-delay cycle $p_{1}, p_{5}, p_{3}$, due to the existence of more than one set of assumptions for $p_{1}$ it is possible to generate real proofs. 
Figure 4 shows the pseudo-code for Guarantee. For a

$/ / \mathcal{G}$ is the proof graph

// $C$ is a given set of assumed properties

Algorithm Guarantee $(\mathcal{G}, C)$

$1 \phi_{0}(v)=1, \forall v \in \mathcal{V}$

$2 w=0$

3 while (1)

$4 \quad w=w+1$

$5 \phi_{w}(v)=X, \forall v \in \mathcal{V}$

6 push every vertex into event queue

7 while the event queue is not empty

8 pop vertex $v$ out of the event queue

9 if $v$ is $\tau_{p}$, where $p \in C$

$10 \quad \phi_{w}(v)=1$

11 elsif $v$ is $\tau_{p}$, where $p \in P_{\text {unknown }}$

$12 \phi_{w}(v)=0$

13

14

15

16

17

18

19

20

21

22

23

24

25

26

27

28

29

30 endwhile

elsif $v$ is $\tau_{p}$, where $p \in P_{\text {impld }}$

let $\phi_{w}(v)$ have its input $o_{p}$ 's value

elsif $v$ is an OR vertex

let $\phi_{w}(v)$ be the OR of its inputs' values else

$v$ must be an AND vertex

$\phi_{w}(v)=1$

foreach input edge $e=\left\langle\tau_{q}, v\right\rangle$

if $e \in \mathcal{E}^{+}$

$\phi_{w}(v) \&=\phi_{w-1}\left(\tau_{q}\right)$

else

$\phi_{w}(v) \&=\phi_{w}\left(\tau_{q}\right)$

if $\phi_{w}(v)$ has changed

push all its fanout vertices into event queue

endwhile

if $\phi_{w}==\phi_{w-1}$

return $\left\{p \mid p \in\left(P_{\text {impld }} \backslash C\right) \wedge \phi_{w}\left(\tau_{p}\right)==1\right\}$

Fig. 4. Calculate the guarantees from assumptions

given proof graph $\mathcal{G}$ and a subset $C \subseteq\left(P_{\text {unknown }} \cup P_{\text {impld }}\right)$, it can be shown that $M \models \square C \rightarrow \square$ Guarantee $(\mathcal{G}, C)$. Guarantee $(\mathcal{G}, \emptyset)$ generates real proofs without assumptions. In this algorithm, the possible truth values for a graph vertex are $0,1, X$, where 0 represents false, 1 represents true, and $X$ could be either true or false. We define function $\phi_{w}: \mathcal{V} \rightarrow\{0,1, X\}$ as a mapping from vertices in iteration $w$ (of the code block starting on Line 7 ) to truth values. At iteration $0, \phi_{0}(v)$ is 1 for every vertex $v$. From Line 7 to Line $27, \phi_{w}$ is calculated using event driven simulation. The code between Line 20 to Line 24 warrants detailed explanation. For a unit-delay assumption, values for the previous iteration are used; otherwise, values from the current iteration are used. Using lattice $1 \succeq X \succeq 0$, it is possible to prove that this algorithm performs a greatest fixed point computation $\langle 1, \ldots, 1\rangle=\phi_{0} \succeq \phi_{1} \succeq \cdots \succeq \phi_{\infty}$. When the fixed point is reached (Line 28), the set of guaranteed properties is $\left\{p \mid p \in\left(P_{\text {impld }} \backslash C\right) \wedge \phi_{\infty}\left(\tau_{p}\right)==1\right\}$.

\section{Generate Assume Guarantee Report}

For the remaining indeterminate properties, we generate a report that restricts the manual analysis of these properties to a potentially much smaller set of properties. An example of such a report is shown in Figure 5. In this figure, there

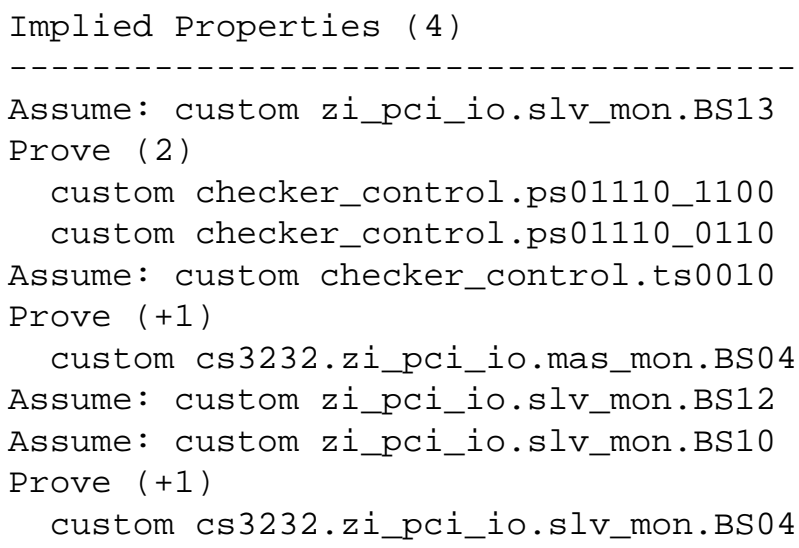

Fig. 5. An assume guarantee report

are four implied properties listed under "Prove". To guarantee these implied properties, there are four assumptions whose correctness need to be established. If the first of the four assumptions is proven, two (i.e. 2 in Figure 5) implied properties are guaranteed to be correct. If the second assumption is proven, one more (i.e. the first +1 in Figure 5) implied property is guaranteed to be correct. Finally, if the last two assumptions are proven, one more (i.e. the last +1 in Figure 5) implied property is guaranteed to be correct. Note that the last property requires all the four assumptions in the table. Using such a report, users can follow the suggested order to discharge all the required assumptions. For users who wish to see the assumptions for each implied property listed separately, we also provide the detailed assume guarantee relations.

Figure 6 shows the pseudo-code for Guide to generate an assume guarantee report. In this figure, $C$ is the set of selected assumptions and $G$ is the set of implied assertions that can be guaranteed by $C$. Between Line 2 and Line 9 , assumptions are identified and propagated using Guarantee until all implied properties are guaranteed. $R$ associates each assumption with the number of properties that become guaranteed. At Line 10, $R$ is sorted so that assumptions with more guaranteed properties are ordered first. The assumptions and their guarantees are then printed between Line 12 and Line 17 . The DFS algorithm at Line 4 finds an assertion $q$ in the transitive fanin of $\tau_{p}$ that is not in $C \cup G$. It is easy to see that by adding $q$ into $C, p$ is closer to being guaranteed. $C$ can include both unknown properties and implied properties. Note that it is also possible to improve the ordering of assumptions by iterating this algorithm more than once. 
$/ / \mathcal{G}$ is the proof graph

Algorithm Guide $(\mathcal{G})$

$1 C=G=\emptyset$

2 while $\left((C \cup G) \nsupseteq P_{\text {impld }}\right)$

3 pick any $p \in P_{\text {impld }} \backslash(C \cup G)$

$4 \tau_{q}=\mathrm{DFS}\left(\mathcal{G}, \tau_{p},(C \cup G)\right)$

$5 \quad C=C \cup q$

$6 \quad N=$ Guarantee $(\mathcal{G}, C)$

$7 \quad R=R \cup\{\langle q,|N|-|G|\rangle\}$

$8 \quad G=N$

9 endwhile

10 sort $R$ in descending order of the second elements

$11 C=G=\emptyset$

12 foreach $(\langle q, n\rangle \in R)$

$13 \quad C=C \cup q$

$14 \quad N=$ Guarantee $(\mathcal{G}, C)$

15 print $(q, N \backslash G)$

$16 \quad G=N$

17 endfor

Fig. 6. Algorithm to generate an assume guarantee report

\section{EXPERIMENTS}

In this section, we report experimental results for eight industrial designs of various sizes and complexities. The largest design has over 135k registers and 900k gates. The smallest design has 600 registers and $2 \mathrm{k}$ gates. The numbers of assertions per design range from 82 to $6 \mathrm{k}$. Our experiments were run on a $3.2 \mathrm{Ghz}$ Pentium4 with $3.4 \mathrm{~GB}$ memory running RedHat Linux 7.2. Table II shows the results for designs D1 to D8. The second and third columns contain the number of register and assertion for each design. Columns 4 and 5 compare the number of assertions that remain for further analysis (1) using and (2) without using our automatic assume guarantee algorithms respectively. When the algorithms in this paper are disabled, we report the number of unknown properties; otherwise, we report the sum of the unknown properties and the number of assumptions (identified by Algorithm Guide in Section V). Based on this table, automatic assume guarantee can reduce the number of assertions remaining for manual analysis by $(899-664) / 899=26 \%$ on average. This represents a significant saving of users' time and effort. Columns 6 and 7 compare the runtime (1) using and (2) without using assume guarantee. The overhead of our assume guarantee algorithms is pretty low. The average slowdown is $(6763-5976) / 5976=13 \%$. The last column reports the number of real proofs computed by the Guarantee algorithm in Section IV. Note that except for 1 proof in design D4 and 2 proofs in design D5, these proofs are also proven without using assume guarantee. However, finding these proofs through direct brute force methods was typically much more computationally expensive than using our assume guarantee reasoning and leveraging proofs already found for other properties. This in part explains the low overhead of our assume guarantee algorithms, despite the amount of analysis they perform.

\begin{tabular}{|l|r|r|r|r|r|r|r|}
\hline Design & Reg & Prop & \multicolumn{2}{|c|}{ Remaining } & \multicolumn{2}{|c|}{ Time (secs) } & Proof \\
\cline { 4 - 7 } & & & With & W/O & With & W/O & \\
\hline \hline D1 & $2 \mathrm{k}$ & 363 & 35 & 150 & 56 & 52 & 14 \\
\hline D2 & $50 \mathrm{k}$ & 219 & 50 & 74 & 795 & 532 & 0 \\
\hline D3 & $68 \mathrm{k}$ & 739 & 93 & 126 & 2472 & 2296 & 46 \\
\hline D4 & $55 \mathrm{k}$ & 6476 & 40 & 54 & 1475 & 1407 & $1 / 96$ \\
\hline D5 & $65 \mathrm{k}$ & 375 & 215 & 245 & 571 & 367 & $2 / 3$ \\
\hline D6 & $17 \mathrm{k}$ & 132 & 79 & 87 & 71 & 43 & 1 \\
\hline D7 & $135 \mathrm{k}$ & 418 & 101 & 109 & 1212 & 1169 & 15 \\
\hline D8 & 600 & 82 & 51 & 54 & 111 & 110 & 0 \\
\hline \hline Total & & 8804 & 664 & 899 & 6763 & 5976 & $3 / 175$ \\
\hline
\end{tabular}

TABLE II

EXPERIMENTS ON EIGHT INDUSTRIAL DESIGNS.

\section{CONCLUSIONS}

We have presented automatic assume guarantee algorithms for assertion based formal verification. Our algorithms exploit the large number of assertions in an $\mathrm{ABV}$ methodology to automatically compute the assume guarantee relations. Experiments with industrial designs demonstrate that a significant number of assertions can be excluded from manual analysis. Our approach is easily introduced into an existing ABV methodology. No manually supplied assume guarantee relations are required. Users are provided with an ordered list of assertions to analyze. Users do not need to know the detailed assume guarantee relations.

\section{REFERENCES}

[1] R. Alur, L. Alfaro, T. Henzinger, and F. Mang, "Automating modular verification," in CONCUR, 1999, pp. 82-97.

[2] A. Biere, A. Cimatti, E. Clarke, and Y. Zhu, "Symbolic model checking without BDDs," in TACAS, 1999.

[3] P. Chauhan, E. Clarke, S. Sapra, J. Kukula, H. Veith, and D. Wang, "Automated abstraction refinement for model checking large state spaces using sat based conflict analysis," in FMCAD, 2002.

[4] J. Cobleigh, D. Giannakopoulou, and C. Pasareanu, "Learning assumptions for compsotional verification," in TACAS, 2003.

[5] N. Een and N. Sorensson, "Temporal induction by incremental sat solving," in First International Workshop on Bounded Model Checking, 2003.

[6] A. Gupta, M. Ganai, P. Ashar, and Z. Yang, "Iterative abstraction using sat-based bmc with proof analysis," in ICCAD, 2003.

[7] T. Henzinger, S. Qadeer, and S. Rajamani, "You assume, we guarantee: methodology and case studies," in CAV, 1998, pp. 440-451.

[8] K. McMillan and N. Amla, "Automatic abstraction without counterexamples," in TACAS, 2003.

[9] K. L. McMillan, "Circular compositional reasonining about liveness," in CHARME, 1999.

[10] A. Pnueli, "In transition from global to modular temporal reasoning about programs," in Logic and Models of Concurrent Systems, 1984, pp. 123-144.

[11] M. Sheeran, S. Singh, and G. Stalmarck, "Checking safety properties using induction and a sat-solver," in FMCAD, 2000.

[12] D. Wang, P.-H. Ho, J. Long, J. Kukula, Y. Zhu, T. Ma, and R. Damiano, "Formal Property Verification by Abstraction Refinement with Formal, Simulation and Hybrid Engines," in DAC, 2001. 